\title{
Growth rates of the Weibel and tearing mode instabilities in a relativistic pair plasma
}

\author{
J Pétri and J G Kirk \\ Max-Planck-Institut für Kernphysik, Saupfercheckweg 1, 69117 Heidelberg, Germany \\ E-mail: j.petri@mpi-hd.mpg.de
}

\begin{abstract}
We present an algorithm for solving the linear dispersion relation in an inhomogeneous, magnetised, relativistic plasma. The method is a generalisation of a previously reported algorithm that was limited to the homogeneous case. The extension involves projecting the spatial dependence of the perturbations onto a set of basis functions that satisfy the boundary conditions (spectral Galerkin method). To test this algorithm in the homogeneous case, we derive an analytical expression for the growth rate of the Weibel instability for a relativistic Maxwellian distribution and compare it with the numerical results. In the inhomogeneous case, we present solutions of the dispersion relation for the relativistic tearing mode, making no assumption about the thickness of the current sheet, and check the numerical method against the analytical expression.
\end{abstract}

PACS numbers: 52.27.Ny; 52.35.-g; 95.30.Qd

Submitted to: Plasma Phys. Control. Fusion 


\section{Introduction}

Dissipation of the energy carried by relativistic plasma outflows is important for the physics of pulsar winds and gamma-ray bursts (for recent reviews see [1] and [2]). In these objects, the plasma is probably composed of electrons, positrons and protons. As well as being in relativistic bulk motion with respect to the observer, the random thermal energy of the plasma may also be relativistic, i.e., comparable to the rest mass energy of the constituent particles. In this paper we concentrate on two instabilities: these are the two-stream or Weibel instability [3] and the tearing modes in a relativistic neutral pair plasma current sheet, thought to play a role in the formation process of relativistic shocks [4] and the dissipation of magnetic energy in pulsar winds [5]. They are investigated by generalising and extending to the inhomogeneous case the method presented in [6]. Motivated primarily by the need for code verification, we have derived some analytical expressions for the linear growth rates of these instabilities.

The Weibel instability is very important in astrophysical processes because it is able to generate a magnetic field by extracting the free energy from an anisotropic momentum distribution in an unmagnetised plasma or from the kinetic drift energy. There is an extensive literature on the Weibel instability: general conditions for the existence of the relativistic Weibel instability for arbitrary distribution functions are discussed in [7], and wave propagation in counter-streaming magnetised nonrelativistic Maxwellian plasmas are studied in [8, 9]. Dispersion curves have been found in some special cases such as, for example, the fully relativistic bi-Maxwellian distribution function, (Yoon [12]), and the water-bag distribution function, in which case closed-form analytical expressions can be derived not only for the Weibel instability (Yoon [10]), but also for the cyclotron maser and whistler instabilities (Yoon [11]). However, finding an analytical expression for the dispersion relation for a given equilibrium distribution function is a complicated or even impossible task. It involves a four-dimensional integration (3D in momentum space and $1 \mathrm{D}$ in time) of the equilibrium distribution function which is difficult to perform in closed form. For this reason, the water-bag distribution is the preferred profile to analyse magnetic field generation in fast ignitor scenarios, (Silva et al. [13]) and in relativistic shocks, (Wiersma and Achterberg [14], Lyubarsky and Eichler [15]). The Weibel instability in a magnetised electron-positron pair plasma has been investigated by Yang et al [16] using two model distributions: the water bag, and one with a powerlaw dependence at high energy. A general covariant description has been formulated by Melrose [17] and by Schlickeiser [18]. In the present work, we focus on equilibrium configurations given by a relativistic Maxwellian distribution function, which allows one to reduce the four-dimensional integral to a simple one-dimensional integral, as we demonstrate in Section 2.2. The growth rates are then found by solving this equation using a single numerical quadrature, and are compared to the results found using our extended algorithm in Section 4.1.

In the inhomogeneous case, the stability properties of a nonrelativistic Harris current sheet also have a substantial literature, with notable recent studies by 
Daughton [19] and Silin et al. [20]. In the relativistic case, the tearing mode instability has been investigated by Zelenyi \& Krasnoselskikh [21], by integrating first order perturbations of the relativistic Maxwellian distribution function along approximate, straight-line particle trajectories, in the thick layer limit (in which the Larmor radius is much smaller than the thickness of the current sheet). In Section 2.4 we lift these restrictions to present new results for the tearing mode instability in a neutral current sheet of arbitrary temperature and thickness, and compare these with the results found using the generalised algorithm in Section 4.2. In this work, no assumption is made about the thickness of the current sheet, and the particle trajectories are found numerically in the background magnetic field.

The numerical method, which is an extension of our previous algorithm, [6], that computes the linear dispersion relation of waves within a Vlasov-Maxwell description, is described in Section 3. It is based on the approach of Daughton [19] for non relativistic Maxwellians, and involves explicit time integration of particle orbits along the unperturbed trajectories. We modify and extend our former code to include inhomogeneities in the plasma equilibrium configuration. Moreover, we generalise it to a fully relativistic approach, i.e., one that allows for relativistic temperatures as well as relativistic drift speeds.

\section{Analytical treatment of the instabilities}

\subsection{The Vlasov-Maxwell equations}

For convenience, we first recall the full set of Vlasov-Maxwell equations governing the non-linear time evolution of the pair plasma.

We introduce the standard electromagnetic scalar and vector potentials $(\phi, \vec{A})$, related to the electromagnetic field $(\vec{E}, \vec{B})$ by :

$$
\begin{aligned}
\vec{E} & =-\vec{\nabla} \phi-\frac{\partial \vec{A}}{\partial t} \\
\vec{B} & =\vec{\nabla} \wedge \vec{A}
\end{aligned}
$$

We employ the Lorenz gauge condition by imposing

$$
\operatorname{div} \vec{A}+\varepsilon_{0} \mu_{0} \frac{\partial \phi}{\partial t}=0
$$

with $\varepsilon_{0} \mu_{0} c^{2}=1$ and $c$ the speed of light. The relation between potentials and sources then reads :

$$
\begin{aligned}
& \Delta \phi-\frac{1}{c^{2}} \frac{\partial^{2} \phi}{\partial t^{2}}+\frac{\rho}{\varepsilon_{0}}=0 \\
& \Delta \vec{A}-\frac{1}{c^{2}} \frac{\partial^{2} \vec{A}}{\partial t^{2}}+\mu_{0} \vec{j}=0
\end{aligned}
$$

The source terms represented by the charge $\rho$ and current $\vec{j}$ densities, are expressed in 
terms of the distribution functions of each species, $f_{s}$, by :

$$
\begin{aligned}
& \rho(\vec{r}, t)=\sum_{s} q_{s} \iiint f_{s}(\vec{r}, \vec{p}, t) \mathrm{d}^{3} \vec{p} \\
& \vec{j}(\vec{r}, t)=\sum_{s} q_{s} \iiint \frac{\vec{p}}{\gamma m_{s}} f_{s}(\vec{r}, \vec{p}, t) \mathrm{d}^{3} \vec{p}
\end{aligned}
$$

where $\gamma=\sqrt{1+\vec{p}^{2} / m_{s}^{2} c^{2}}$ is the Lorentz factor of a particle. We adopted the usual notations, namely $\left(t, \vec{r}, \vec{v}, \vec{p}, m_{s}, q_{s}\right)$ for respectively the time, position, 3-velocity, 3momentum, mass and charge of a particle of species $s$. The time evolution of the distribution functions $f_{s}$ is governed by a relativistic Vlasov equation for each species:

$$
\frac{\partial f_{s}}{\partial t}+\vec{v} \cdot \frac{\partial f_{s}}{\partial \vec{r}}+q_{s}(\vec{E}+\vec{v} \wedge \vec{B}) \cdot \frac{\partial f_{s}}{\partial \vec{p}}=0
$$

The self-consistent non-linear evolution of the plasma is entirely determined by the set of equations (1)-(6).

The linear stability properties of the neutral current sheet are investigated by linearising the set of equations (11)-(6). The procedure has been described in [6], Sec. 3 . These results are now used to derive linear dispersion relations.

\subsection{Weibel instability}

In the Weibel regime, the electrostatic potential $\phi$ can be neglected. Moreover, only the $A_{\mathrm{y}}$ component of the vector potential $\vec{A}$ comes into play in the eigenvalue problem. Note also that the Weibel instability corresponds to low-frequency modes propagating along the $z$-axis such that

$$
\|\omega\| \ll k_{\mathrm{z}} c
$$

with $k_{\mathrm{y}}=0, \vec{k}=\left(0, k_{\mathrm{y}}, k_{\mathrm{z}}\right)$ being the wavenumber and $\omega$ the corresponding eigenfrequency.

Specialising the general derivation of the perturbed charge density and current density presented in Sect. 3 of [6] to the Weibel instability, setting $\phi=A_{\mathrm{x}}=A_{\mathrm{z}}=k_{\mathrm{y}}=$ 0 , the dispersion relation reads

$$
\begin{aligned}
& \frac{\omega^{2}}{c^{2}}-k_{\mathrm{z}}^{2}+\frac{2 \Gamma_{s}^{2} \beta_{s}^{2} \omega_{\mathrm{p} s}^{2}}{c^{2} \Theta_{s}}+\sum_{s} i \omega \iiint \frac{f_{0 s} p_{\mathrm{y}}}{\gamma m_{s}^{2}} \times \\
& \times \int_{-\infty}^{0} p_{\mathrm{y}}^{\prime} \exp \left[i\left(k_{\mathrm{z}} p_{\mathrm{z}} / m_{s}-\gamma \omega\right) \tau^{\prime}\right] d \tau^{\prime} d^{3} \vec{p}=0
\end{aligned}
$$

The distribution function at equilibrium for each species " $s$ ", denoted by $f_{0 s}(\vec{r}, \vec{p})$, is assumed to be a relativistic Maxwellian with constant drift speed $\pm U_{s}$. The stationary distribution function for each species reads:

$$
f_{0 s}(\vec{r}, \vec{p})=\frac{N_{s}}{4 \pi m_{s}^{3} c^{3} \Theta_{s} K_{2}\left(1 / \Theta_{s}\right)} \exp \left[-\Gamma_{s}\left(E-U_{s} p_{\mathrm{y}}\right) / \Theta_{s} m_{s} c^{2}\right]
$$

$N_{s}$ is the (constant) particle number density of the plasma, $\omega_{\mathrm{p} s}=N_{s} q_{s}^{2} / m_{s} \varepsilon_{0}$ the plasma frequency, $E=m_{s} c^{2} \sqrt{1+\vec{p}^{2} / m_{s}^{2} c^{2}}$ the total energy of a particle, $p_{\mathrm{y}}$ the y-component 
of its momentum, $\Gamma_{s}=1 / \sqrt{1-\beta_{s}^{2}}\left(\beta_{s}=U_{s} / c\right)$ the Lorentz factor associated with the drift motion and $K_{2}$ the modified Bessel function of the second kind and of order 2 . The temperature of the gas $T_{s}$ is conveniently normalised to the rest mass energy of the leptons such that

$$
\Theta_{s}=\frac{k_{B} T_{s}}{m_{s} c^{2}}
$$

and $k_{B}$ is the Boltzmann constant. The triple integral along the momentum vector can be performed analytically in the following way.

Because no external background electromagnetic field exists at equilibrium, the particle trajectories are straight lines. The integration of the equations of motion leads to

$$
\begin{aligned}
& \vec{p}^{\prime}=\vec{p}=\mathrm{cst} \\
& \vec{r}^{\prime}=\vec{r}+\frac{\vec{p}}{m_{s}} \tau^{\prime}
\end{aligned}
$$

Therefore time and momentum integration can be inverted. The dispersion relation thus reduces to

$$
\begin{aligned}
& \frac{\omega^{2}}{c^{2}}-k_{\mathrm{z}}^{2}+\frac{2 \Gamma_{s}^{2} \beta_{s}^{2} \omega_{\mathrm{p} s}^{2}}{c^{2} \Theta_{s}}+\sum_{s} i \omega \frac{\Gamma_{s} \omega_{\mathrm{p} s}^{2}}{c^{4} \Theta_{s}} \int_{-\infty}^{0} \iiint \frac{p_{\mathrm{y}}^{2}}{\gamma m_{s}^{2}} \times \\
& \times \frac{\exp \left(-\Gamma_{s}\left(E-U_{s} p_{\mathrm{y}}\right) / \Theta_{s} m_{s} c^{2}\right)}{4 \pi m_{s}^{3} c^{3} \Theta_{s} K_{2}\left(1 / \Theta_{s}\right)} \exp \left[i\left(k_{\mathrm{z}} p_{\mathrm{z}} / m_{s}-\gamma \omega\right) \tau^{\prime}\right] d^{3} \vec{p} d \tau^{\prime}=0
\end{aligned}
$$

The integration along the momentum $\vec{p}$ can be done analytically with help on the following formula, see for instance 22 ]

$$
I(A, \vec{\alpha}) \equiv \frac{1}{4 \pi} \iiint \frac{\exp (-A \gamma \pm i \vec{\alpha} \cdot \vec{p})}{\gamma} d^{3} \vec{p}=m_{s}^{3} c^{3} \frac{K_{1}\left(\sqrt{A^{2}+m_{s}^{2} c^{2} \alpha^{2}}\right)}{\sqrt{A^{2}+m_{s}^{2} c^{2} \alpha^{2}}}
$$

where the Lorentz factor of a particle is $\gamma=\sqrt{1+\vec{p}^{2} / m_{s}^{2} c^{2}}$ and $K_{1}$ is the modified Bessel function of order 1 . By differentiating twice with respect to the $y$ component, $\alpha_{\mathrm{y}}$, of the vector $\vec{\alpha}$, we get

$$
\begin{aligned}
I_{\mathrm{py}}(A, \vec{\alpha}) \equiv & -\frac{\partial^{2} I}{\partial \alpha_{\mathrm{y}}^{2}}(A, \vec{\alpha})=\frac{1}{4 \pi} \iiint \frac{p_{\mathrm{y}}^{2} \exp (-A \gamma \pm i \vec{\alpha} \cdot \vec{p})}{\gamma} d^{3} \vec{p}= \\
& m_{s}^{5} c^{5}\left[\frac{A^{2}+m_{s}^{2} c^{2} \alpha^{2}-4 m_{s}^{2} c^{2} \alpha_{\mathrm{y}}^{2}}{\left(A^{2}+m_{s}^{2} c^{2} \alpha^{2}\right)^{2}} K_{2}\left(\sqrt{A^{2}+m_{s}^{2} c^{2} \alpha^{2}}\right)-\right. \\
& \left.\frac{m_{s}^{2} c^{2} \alpha_{\mathrm{y}}^{2}}{\left(A^{2}+m_{s}^{2} c^{2} \alpha^{2}\right)^{3 / 2}} K_{1}\left(\sqrt{A^{2}+m_{s}^{2} c^{2} \alpha^{2}}\right)\right]
\end{aligned}
$$

Applying these formulae to our problem, it is convenient to introduce the following quantities

$$
\begin{aligned}
& A(\omega, \tau)=\frac{\Gamma_{s}}{\Theta_{s}}+i \omega \tau \\
& \vec{\alpha}(\tau)=-i \frac{\Gamma_{s} \beta_{s}}{\Theta_{s} m_{s} c} \vec{e}_{\mathrm{y}}+\frac{k_{\mathrm{z}} \tau}{m_{s}} \vec{e}_{\mathrm{z}}
\end{aligned}
$$


The function $I_{\text {py }}$ depends now on $\omega$ and $\tau$ via $A(\omega, \tau)$ and $\vec{\alpha}(\tau), I_{\text {py }}(A(\omega, \tau), \vec{\alpha}(\tau))$, assuming that the equilibrium quantities such as species temperatures $\Theta_{s}$ and drift speeds $\beta_{s}$ are prescribed and therefore constant. In the remainder of this section, we will denote it for simplicity by $I_{\mathrm{py}}(\omega, \tau)$. The dispersion relation for the Weibel instability, Eq. (13) therefore reads

$$
\frac{\omega^{2}}{c^{2}}-k_{\mathrm{z}}^{2}+\frac{2 \Gamma_{s}^{2} \beta_{s}^{2} \omega_{\mathrm{p} s}^{2}}{c^{2} \Theta_{s}}+\frac{2 i \omega \Gamma_{s} \omega_{\mathrm{p} s}^{2}}{c^{2} \Theta_{s}^{2} K_{2}\left(1 / \Theta_{s}\right)} \frac{1}{m_{s}^{5} c^{5}} \int_{-\infty}^{0} I_{\mathrm{py}}(\omega, \tau) d \tau=0
$$

In the non-relativistic limit, $\Theta_{s} \ll 1$, we recover the dispersion relation Eq.(33) of [6].

\subsection{Relativistic neutral current sheet}

We now turn to the study of the unstable tearing mode in a relativistic neutral current sheet of thickness $L$ and asymptotic magnetic field intensity $B_{0}$ (far from the current sheet). The plasma is one-dimensional in the sense that it has only spatial variation in the $x$ direction. It consists of counter-streaming electrons and positrons with relativistic temperatures $T_{s}$ evolving in a static external magnetic field aligned with the $z$-axis such that [23]

$$
B_{\mathrm{z}}(\vec{r})=B_{0} \tanh \left(\frac{x}{L}\right)
$$

We use Cartesian coordinates, denoted by $\vec{r}=(x, y, z)$, and the corresponding basis $\left(\vec{e}_{\mathrm{x}}, \vec{e}_{\mathrm{y}}, \vec{e}_{\mathrm{z}}\right)$. In equilibrium, there is no electric field, $\vec{E}_{0}=\overrightarrow{0}$ and the charges drift in the $y$ direction at a relativistic velocity $U_{s}$. The particle number density for each species is

$$
n_{0 s}(\vec{r})=N_{s} \operatorname{sech}^{2}\left(\frac{x}{L}\right)
$$

The distribution function at equilibrium for each species " $s$ ", denoted by $f_{0 s}(\vec{r}, \vec{p})$, is assumed to be a relativistic Maxwellian with constant drift speed $\pm U_{s}$. The stationary distribution function for each species reads:

$$
f_{0 s}(\vec{r}, \vec{p})=\frac{n_{0 s}(\vec{r})}{4 \pi m_{s}^{3} c^{3} \Theta_{s} K_{2}\left(1 / \Theta_{s}\right)} \exp \left[-\Gamma_{s}\left(E-U_{s} p_{\mathrm{y}}\right) / \Theta_{s} m_{s} c^{2}\right]
$$

$n_{0 s}(\vec{r})$ is the particle number density in the current sheet, Eq. (20), and all other quantities are the same as those defined for the distribution function in Eq. (9). The stationary Vlasov-Maxwell equations are satisfied provided that

$$
\begin{aligned}
4 N_{s} \Theta_{s} m_{s} c^{2} & =\frac{B_{0}^{2}}{\mu_{0}} \\
\Gamma_{s} U_{s} & =-2 \frac{\Theta_{s} m_{s} c^{2}}{q_{s} B_{0} L}
\end{aligned}
$$

Eq. (22) states the balance between gaseous pressure and magnetic pressure at the centre

of the current sheet. It is also useful to note that the non-relativistic plasma frequency (at the centre of the current sheet) and cyclotron frequency defined, respectively, by

$$
\hat{\omega}_{p s}^{2}=\frac{N_{s} q_{s}^{2}}{m_{s} \varepsilon_{0}}
$$




$$
\hat{\Omega}_{B s}=\frac{q_{s} B_{0}}{m_{s}}
$$

are related by

$$
4 \hat{\omega}_{p s}^{2} \Theta_{s}=\hat{\Omega}_{B s}^{2}
$$

These frequencies are constant (independent of $x$ ), and are denoted by a ^ in order to distinguish them from quantities that depend on $x$.

\subsection{The relativistic tearing mode}

A method similar to that used in Section 2.2 can also be applied to the relativistic neutral current sheet. Using the equilibrium distribution function presented in Eq. (21) for the relativistic Harris current sheet, the eigenvalue equation to solve reads

$$
\begin{aligned}
& A_{\mathrm{y}}^{\prime \prime}(x)-\left(k_{\mathrm{z}}^{2}-\frac{\omega^{2}}{c^{2}}\right) A_{\mathrm{y}}(x)+\frac{2}{L^{2}} \operatorname{sech}^{2}\left(\frac{x}{L}\right) A_{\mathrm{y}}(x)+ \\
& \frac{2 i \omega \Gamma_{s} \hat{\omega}_{\mathrm{p} s}^{2}}{c^{2} \Theta_{s}^{2} K_{2}\left(1 / \Theta_{s}\right)} \frac{A_{\mathrm{y}}(x)}{m_{s}^{5} c^{5}} \int_{-\infty}^{0} I_{\mathrm{py}}(\omega, \tau) d \tau=0
\end{aligned}
$$

where prime " denotes second derivative with respect to $x$. We now follow [24] and introduce the variable $t=\tanh (x / L)$, which transforms Eq. (27) into the Legendre equation. From this, one sees that the dispersion relation is satisfied by purely growing modes, $\operatorname{Re}(\omega)=0$, the tearing modes, whose growth rate is the solution of

$$
\frac{2 i \omega \Gamma_{s} \hat{\omega}_{\mathrm{p} s}^{2} L^{2}}{c^{2} \Theta_{s}^{2} K_{2}\left(1 / \Theta_{s}\right)} \frac{1}{m_{s}^{5} c^{5}} \int_{-\infty}^{0} I_{\mathrm{py}}(\omega, \tau) d \tau=\left(k_{\mathrm{z}} L+2\right)\left(k_{\mathrm{z}} L-1\right)
$$

where we used the "low-frequency" approximation, Eq. (7), to neglect the contribution of the displacement current. Expression (28) contains a single one-dimensional integral. Finding its solutions is computationally much faster than dealing with the general expression in 4 dimensions and does not involve any approximation concerning the current sheet thickness.

In the low-temperature limit, $\Theta_{s} \ll 1$ (non-relativistic case), the dispersion relation Eq. (27) reduces to its classical expression given by

$$
\frac{\omega}{k_{\mathrm{z}} v_{\mathrm{th}}} Z\left(\frac{\omega}{k_{\mathrm{z}} v_{\mathrm{th}}}\right) \sum_{s}\left(1+2 \frac{U_{s}^{2}}{v_{\mathrm{th}}^{2}}\right)=\frac{c^{2}}{\hat{\omega}_{\mathrm{p} s}^{2} L^{2}}\left(k_{\mathrm{z}} L+2\right)\left(k_{\mathrm{z}} L-1\right)
$$

where the plasma dispersion function $Z$ is defined for $\operatorname{Im}(\zeta)>0$ by

$$
Z(\zeta)=\frac{1}{\sqrt{\pi}} \int_{-\infty}^{+\infty} \frac{\mathrm{e}^{-t^{2}}}{t-\zeta} \mathrm{d} t
$$

and analytically continued for $\operatorname{Im}(\zeta)<0$, see for instance Delcroix and Bers [25].

\section{The algorithm}

We first recall the general linear eigenvalue system to be solved, as presented in [6] and then present the extended algorithm for inhomogeneous and magnetised plasmas. 


\subsection{The eigenvalue system}

The eigenvalue system is found by solving the equations for the electromagnetic potential determined according to the source distribution given by $(5 a b)$ and $(5 b)$. Inserting the latter expressions into (4a) and (4b), the eigenvalue system reads :

$$
\begin{aligned}
& \phi^{\prime \prime}(x)-\left(\vec{k}^{2}-\frac{\omega^{2}}{c^{2}}\right) \phi(x)+\frac{\rho(x)}{\varepsilon_{0}}=0 \\
& \vec{A}^{\prime \prime}(x)-\left(\vec{k}^{2}-\frac{\omega^{2}}{c^{2}}\right) \vec{A}(x)+\mu_{0} \vec{j}(x)=0
\end{aligned}
$$

For homogeneous plasmas, the system reduces to a $4 \times 4$ matrix, which is easily solved, see [6]. In the inhomogeneous case, the perturbations should vanish asymptotically, when $x= \pm \infty$. To solve this inhomogeneous eigenvalue problem, it is convenient to expand the unknown quantities on a set of basis functions, each function individually satisfying the required boundary conditions. This method is known as a spectral Galerkin method, similar to Fourier decomposition for periodic boundary conditions. We will refer to it as a spectral decomposition as described below.

\subsection{Spectral decomposition}

The perturbations are projected on an orthonormal set of basis functions, $\mathcal{F}_{n}$. Because of the required boundary conditions, namely $\phi(x= \pm \infty)=0, \vec{A}(x= \pm \infty)=0$, the Hermite functions are a convenient set onto which to expand the perturbations, [19]. They are given by

$$
\mathcal{F}_{n}(x)=\frac{H_{n}(x)}{\sqrt{2^{n} n ! \sqrt{\pi}}} \exp \left[-x^{2} / 2\right]
$$

where $H_{n}$ are the Hermite polynomials, [26]. We introduce the projection operator of a function $g$ on a basis function $\mathcal{F}_{n}$ defined as

$$
<g \mid \mathcal{F}_{n}>\equiv \int_{-\infty}^{+\infty} g(x) \mathcal{F}_{n}(x) d x
$$

The basis functions themself satisfy the orthonormality relation

$$
<\mathcal{F}_{n} \mid \mathcal{F}_{k}>=\delta_{n k}
$$

where $\delta_{n k}$ is the kronecker symbol. The matrix representation of the second derivative, useful for projection of Eq. (31a) and ( $(31 b)$, is then represented by

$$
\begin{aligned}
\mathcal{D}_{n k} & \equiv \int_{-\infty}^{+\infty} \mathcal{F}_{n}(x) \mathcal{F}_{k}^{\prime \prime}(x) d x \\
& =\frac{\sqrt{(n+1)(n+2)}}{2} \delta_{n, k+2}-\frac{2 n+1}{2} \delta_{n k}+\frac{\sqrt{n(n-1)}}{2} \delta_{n, k-2}
\end{aligned}
$$

For convenience, we introduce the unknown four dimensional vector $\vec{\Psi}=(\phi, \vec{A})$. Using $N$ terms in the expansion of $\vec{\Psi}$, it is written as

$$
\vec{\Psi}(x)=\sum_{k=0}^{N-1} \vec{\Psi}_{k} \mathcal{F}_{k}(x)
$$


Inserting this expression in Eq. ( $(31 a)$ and $(\underline{31 b})$ and projecting them on each basis function $\mathcal{F}_{n}$, the system reduces to a matrix equation of dimension $4 N \times 4 N$ for the $N$ unknown vectors $\vec{\Psi}_{k}$

$$
M(\omega, \vec{k}) \cdot \vec{\Psi}=0
$$

More explicitly, let $C_{k}$ be the $4 N$ unknowns. We choose to order the unknowns in the following way, $\vec{\Psi}_{k}=\left(C_{k}, C_{k+N}, C_{k+2 N}, C_{k+3 N}\right)$. The expansion Eq. (36) is done such that

$$
\begin{aligned}
& \phi(x)=\sum_{k=0}^{N-1} C_{k} \mathcal{F}_{k}(x) \\
& A_{\mathrm{x}}(x)=\sum_{k=0}^{N-1} C_{k+N} \mathcal{F}_{k}(x) \\
& A_{\mathrm{y}}(x)=\sum_{k=0}^{N-1} C_{k+2 N} \mathcal{F}_{k}(x) \\
& A_{\mathrm{z}}(x)=\sum_{k=0}^{N-1} C_{k+3 N} \mathcal{F}_{k}(x)
\end{aligned}
$$

Eq. (37) is a non-linear eigenvalue problem for the matrix $M$ with eigenvector $\vec{\Psi}$ and eigenvalue $\omega$. The method of solution has been described in [6]. However, the simultaneous search of the eigenvalues and the eigenvectors is very time-consuming because the root finding takes place in a $8 N+2$ dimension parameter space $(4 N+1$ complex numbers to find, the eigenvalue $\omega$ and the $C_{k}$ ). Therefore, we decide only to look for the vanishing of the matrix determinant, $\operatorname{det} M(\omega, \vec{k})=0$. In this paper, we only present the capabilities of our algorithm and do not discuss in detail the physics of the unstable perturbations. For this purpose, it is sufficient to look only for the vanishing of the determinant of the matrix $M$. Nevertheless, we present an example of an eigenfunction in Section 4.2, where we examine the convergence properties of the summations in Eqs. (38a $38 d$ d $)$.

\section{Results}

\subsection{Weibel instability}

As a first check, we compute the dispersion relation for the Weibel instability for arbitrary plasma temperature $\Theta_{s}$. The fully relativistic algorithm is checked against the numerical solution to the exact analytical dispersion relation, Eq. (18). The evolution of the growth rates with increasing plasma temperature at a given drift speed is shown in fig[1. In our previous work, [6], the non-relativistic case was solved with a non-relativistic code whereas in the present work, our algorithm deals with arbitrary temperatures (especially in the limit $\left.\Theta_{s} \ll 1\right) \pm$. In particular, the results of the non-relativistic

$\ddagger$ However, this limit needs special care because of the exponentially decreasing of the Bessel function $K_{2}$ in the expressions Eq. (21), implying numerical under- and overflow problems. 
Weibel instability are recovered with the fully relativistic algorithm. Inspecting fig 1, the solutions of our algorithm (denoted by symbols) are in perfect agreement with the growth rates given by numerically solving Eq. (18) (solid lines), for arbitrary temperature. Due to the spread in momentum present at finite temperature, the instability is suppressed for large wavenumbers $k_{\mathrm{z}}$. More precisely, when the temperature increases, the largest unstable wavenumber decreases. For a given drift speed, the growth rates decrease with increasing temperature.

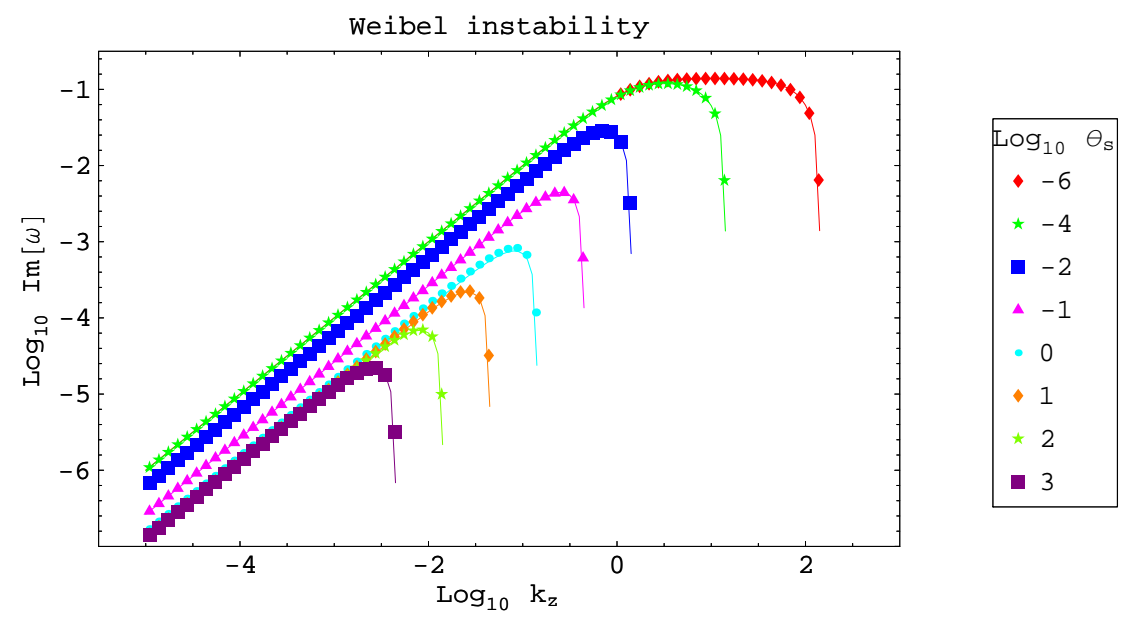

Figure 1. Growth rates of the Weibel instability for different temperatures $\Theta_{s}$, see legend, and a given drift speed $\beta_{s}=0.1$. Points are results from the algorithm and the solid lines are from the numerical solution to the dispersion relation, Eq. (18).

We also investigate the effect of the drift speed on the growth rates for a fixed temperature. Results are shown in the non-relativistic limit $\Theta_{s}=10^{-4}, 10^{-2}$, the mildly relativistic case $\Theta_{s}=1$, and the ultra-relativistic case $\Theta_{s}=10^{2}$, for increasing drift speed $\beta_{s}=0.1 / 0.3 / 0.5$, respectively red diamonds, green stars and blue squares, fig. 2 ,

The characteristic cut-off wavenumber, $k_{\text {cut }}$, can be estimated by setting $\omega=0$ in the dispersion relation Eq. (18). Doing so we find

$$
\frac{k_{\mathrm{cut}} c}{\omega_{\mathrm{p} s}}=\Gamma_{s} \beta_{s} \sqrt{\frac{2}{\Theta_{s}}}
$$

Moreover, in the ultrarelativistic limit, for high temperature $\Theta_{s} \gg 1$ but small drift speeds $\beta_{s} \ll 1$, the growth rates in the small wavenumber limit are simply given by

$$
\gamma_{\mathrm{rel}}=\frac{4}{\pi} \beta_{s}^{2} k_{\mathrm{z}} c
$$

This result is in agreement with the curves shown in fig. 1. Note that these growth rates become independent of the temperature, as in the non-relativistic limit (low temperature), where

$$
\gamma_{\text {clas }}=\beta_{s} k_{\mathrm{z}} c
$$

Therefore, in the ultrarelativistic temperature limit, the growth rates are reduced by a factor $4 \beta_{s} / \pi \approx 1.27 \beta_{s}$. 

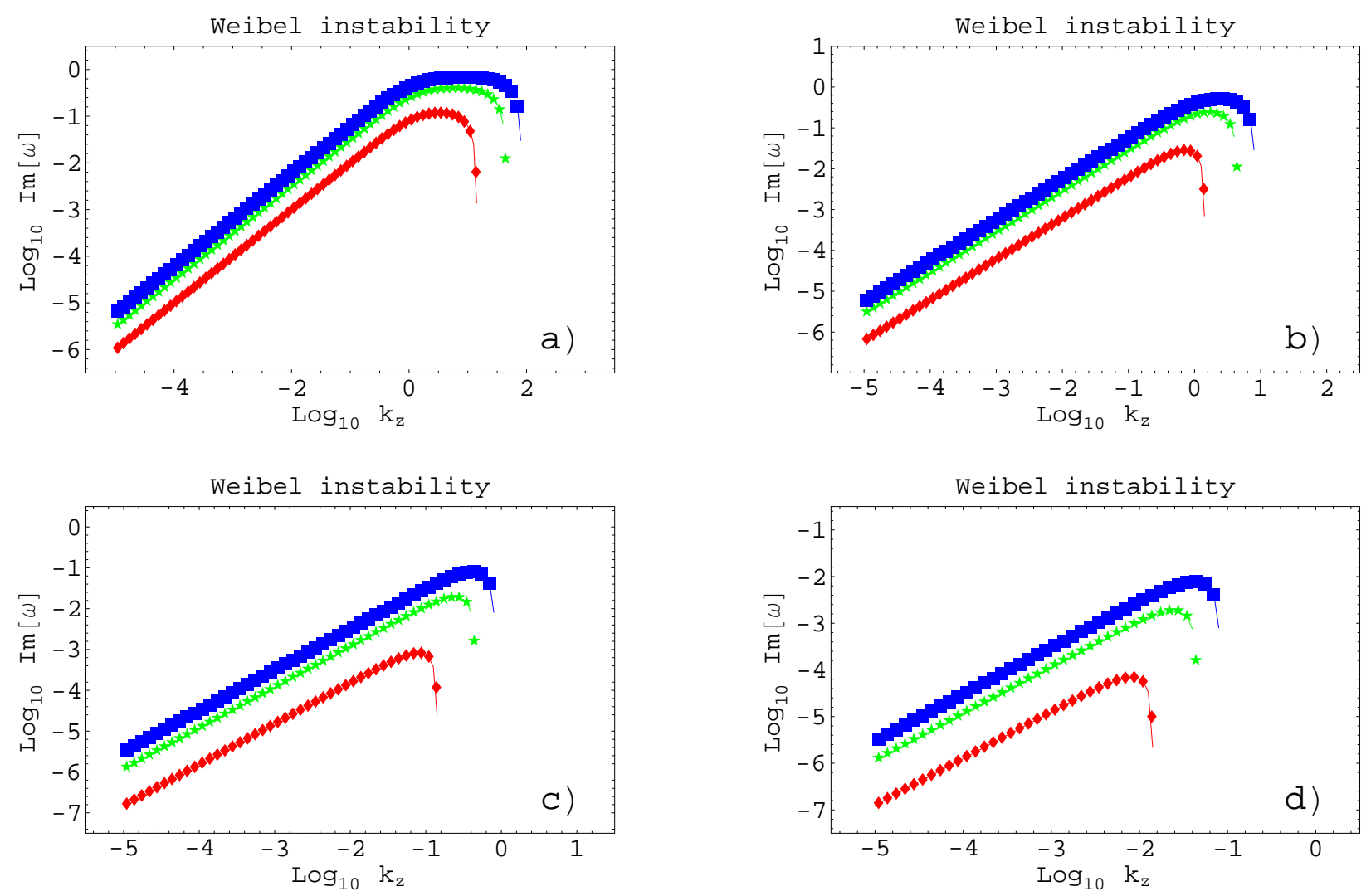

Figure 2. Growth rates of the Weibel instability for different drift speeds $\beta_{s}=$ $0.1 / 0.3 / 0.5$, respectively red diamonds, green stars and blue squares, and a given temperature, $\Theta_{s}=10^{-4}$ in fig a), $\Theta_{s}=10^{-2}$ in fig b), $\Theta_{s}=1$ in fig c), $\Theta_{s}=10^{2}$ in fig d). Points are from our algorithm whereas the solid lines are from the numerical solution to the dispersion relation, Eq. (18).

\subsection{Tearing mode}

Next, we check our algorithm against the tearing mode instabilities in the relativistic Harris current sheet. The eigenvalue problem for the non-relativistic Harris current sheet is given by Galeev, chapter 6.2, page 305, in [27]. The dispersion relation for the non-relativistic tearing modes $\left(k_{\mathrm{y}}=0\right)$ is given in [24, 28]. The relativistic generalisation is given by Eq. (28). In figure 3, we compare our numerical results with the analytic expressions found using a simple root finding algorithm from the dispersion relation for tearing modes in order to check the correctness of our algorithm implementation. The growth rates are normalised to the non-relativistic cyclotron frequency $\hat{\Omega}_{\mathrm{B}}=q_{s} B_{0} / m_{s}$. The numerical results in fig 3 are in good agreement with the approximated analytical expression, Eq. (28). In the spectral decomposition method, it is necessary to choose an appropriate number of terms $N$ in the expansion (36) in order to achieve a given accuracy. To do this, we check the convergence properties of our method. In Fig. 4 we show the eigenvalue as a function of $N$, starting with $N=3$ terms and increasing $N$ until the eigenvalue reaches a satisfactory precision (in this case 1\% accuracy). The 


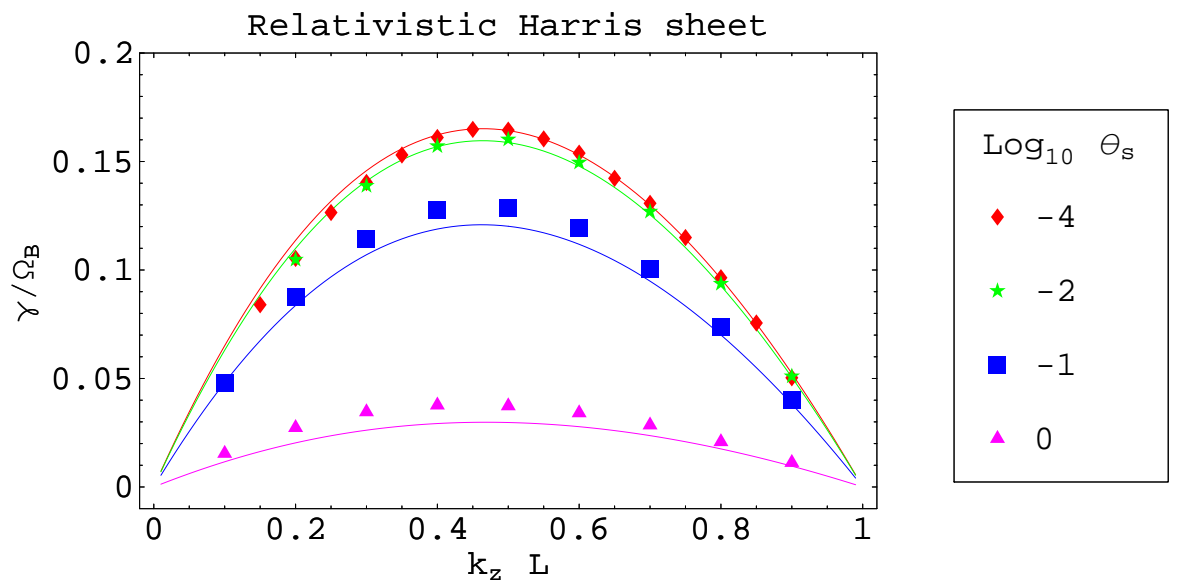

Figure 3. Growth rates of the tearing modes in the Harris current sheet for $\rho=L$. Points are from our numerical algorithm and the solid line represents the analytical approximation in Eq. (28).

corresponding convergence of the eigenfunction is shown in fig 5. Note that the results of fig 3 are shown for $N=11$.

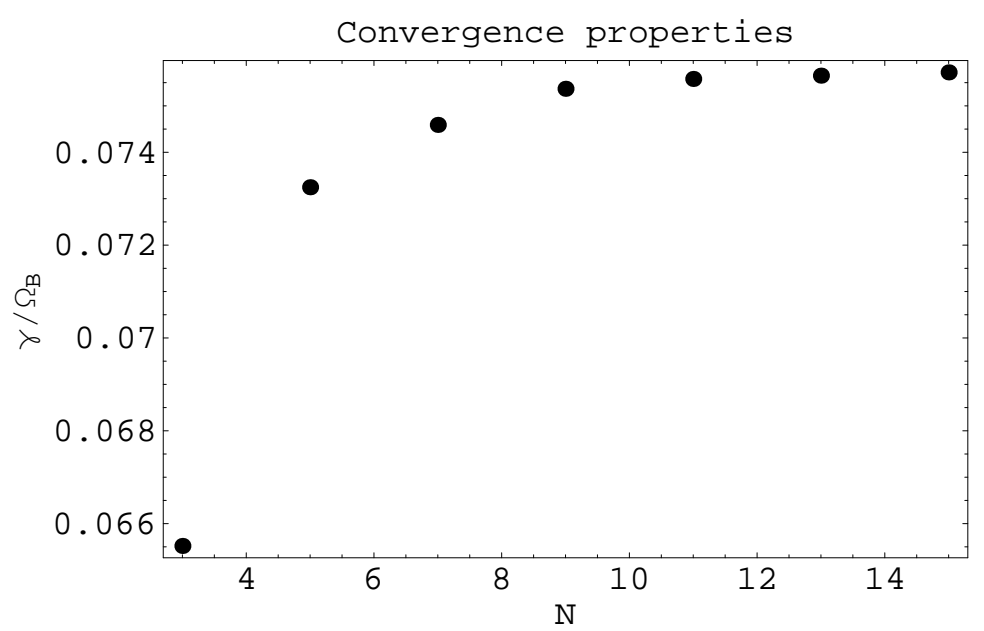

Figure 4. Example of convergence of an eigenvalue when the number of terms in the expansion increases. This specific example corresponds to $k_{\mathrm{z}} L=0.85$ and $\log _{10} \Theta_{s}=-4$ of fig 3 .

\section{Conclusion}

We present a generalisation and extension of our previous algorithm [6] to solve the linear dispersion relation for relativistic multi-component inhomogeneous and magnetised plasmas. The code is validated by comparing the results with two standard configurations: the relativistic Weibel instability in a homogeneous plasma, and the tearing mode instability in a relativistic neutral Harris sheet. To effect the comparison, 


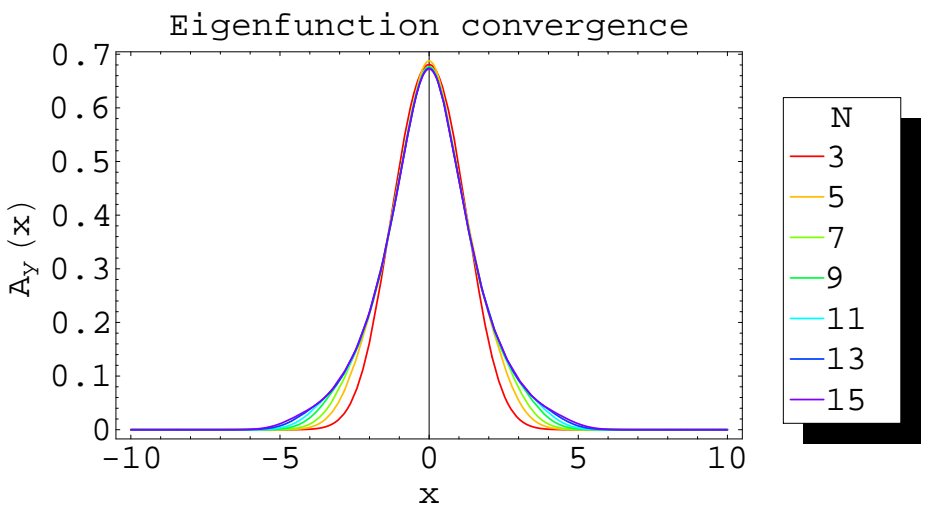

Figure 5. Example of convergence of the eigenfunction associated to the eigenvalue in fig 4. Only the coefficients associated with the even Hermite functions are different from zero as expected from symmetry considerations. The narrowest function corresponds to $N=3$ whereas the widest to $N=15$.

we derived useful analytical expressions, Eq. (18) and Eq. (28), for the dispersion relations in these configurations and solved them numerically. We conclude that this code is a suitable tool for the study of stability properties of more general configurations of interest in gamma-ray burst and pulsar wind theories.

\section{Acknowledgments}

This research was supported by a grant from the G.I.F., the German-Israeli Foundation for Scientific Research and Development.

\section{References}

[1] J. G. Kirk, Y. Lyubarsky, and J. Pétri. The theory of pulsar winds and nebulae. ArXiv Astrophysics e-prints, March 2007.

[2] T. Piran. The physics of gamma-ray bursts. Reviews of Modern Physics, 76:1143-1210, 2005.

[3] E. S. Weibel. Spontaneously Growing Transverse Waves in a Plasma Due to an Anisotropic Velocity Distribution. Physical Review Letters, 2:83-84, February 1959.

[4] A. Spitkovsky. On the structure of relativistic collisionless shocks in electron-ion plasmas. ArXiv e-prints, 706, June 2007.

[5] J. G. Kirk. Relativistic plasmas in pulsar winds. Plasma Physics and Controlled Fusion, 47:B719B726, December 2005.

[6] J. Pétri and J. G. Kirk. Numerical solution of the linear dispersion relation in a relativistic pair plasma. Plasma Physics and Controlled Fusion, 49:297-308, March 2007.

[7] U. Schaefer-Rolffs and R. Schlickeiser. The relativistic kinetic Weibel instability: General arguments and specific illustrations. Physics of Plasmas, 13:2107, 2006.

[8] R. C. Tautz and R. Schlickeiser. Counterstreaming magnetized plasmas. I. Parallel wave propagation. Physics of Plasmas, 12:2901-+, December 2005.

[9] R. C. Tautz and R. Schlickeiser. Counterstreaming magnetized plasmas. II. Perpendicular wave propagation. Physics of Plasmas, 13:2901-+, June 2006.

[10] P. H. Yoon and R. C. Davidson. Exact analytical model of the classical Weibel instability in a relativistic anisotropic plasma. Physical Review A, 35:2718-2721, March 1987. 
[11] P. H. Yoon and R. C. Davidson. Closed-form analytical model of the electron whistler and cyclotron maser instabilities in relativistic plasma with arbitrary energy anisotropy. Physical Review A, 35:2619-2630, March 1987.

[12] P. H. Yoon. Electromagnetic Weibel instability in a fully relativistic bi-Maxwellian plasma. Physics of Fluids B, 1:1336-1338, June 1989.

[13] L. O. Silva, R. A. Fonseca, J. W. Tonge, W. B. Mori, and J. M. Dawson. On the role of the purely transverse Weibel instability in fast ignitor scenarios. Physics of Plasmas, 9:2458-+, June 2002.

[14] J. Wiersma and A. Achterberg. Magnetic field generation in relativistic shocks. An early end of the exponential Weibel instability in electron-proton plasmas. A\&\&A, 428:365-371, December 2004.

[15] Y. Lyubarsky and D. Eichler. Are Gamma-Ray Burst Shocks Mediated by the Weibel Instability? ApJ, 647:1250-1254, August 2006.

[16] T.-Y. B. Yang, Y. Gallant, J. Arons, and A. B. Langdon. Weibel instability in relativistically hot magnetized electron-positron plasmas. Physics of Fluids B, 5:3369-3387, September 1993.

[17] D. B. Melrose. Covariant description of dispersion in a relativistic thermal electron gas. Australian Journal of Physics, 35:41-+, 1982.

[18] R. Schlickeiser. Covariant kinetic dispersion theory of linear waves in anisotropic plasmas. I. General dispersion relation, bi-Maxwellian distribution and non relativistic limits. Physics of Plasmas, 11:5532-+, December 2004.

[19] W. Daughton. The unstable eigenmodes of a neutral sheet. Physics of Plasmas, 6:1329-1343, April 1999.

[20] I. Silin, J. Büchner, and L. Zelenyi. Instabilities of collisionless current sheets: Theory and simulations. Physics of Plasmas, 9:1104-+, April 2002.

[21] L. M. Zelenyi and V. V. Krasnoselskikh. Relativistic Modes of Tearing Instability in a Background Plasma. Soviet Astronomy, 23:460-+, August 1979.

[22] B. A. Trubnikov. Magnetic Emission of High Temperature Plasma. PhD thesis, Dissertation, Moscow (US-AEC Tech. Inf. Service, AEC-tr-4073 [1960]), (1958), 1958.

[23] F. C. Hoh. Stability of sheet pinch. Physics of Fluids, 9:277-284, 1966.

[24] P. L. Pritchett, F. V. Coroniti, R. Pellat, and H. Karimabadi. Collisionless reconnection in twodimensional magnetotail equilibria. J. Geophysical Research, 96:11523-+, July 1991.

[25] J.L. Delcroix and A. Bers. Physique des plasmas - Tome 2. EDP Sciences - CNRS Editions, 1994.

[26] P. M. Morse and H. Feshbach. Methods of theoretical physics. International Series in Pure and Applied Physics, New York: McGraw-Hill, 1953, 1953.

[27] A. A. Galeev and R. Sudan. Basic plasma physics 2. Handbook of Plasma Physics, Amsterdam: North Holland, 1983, edited by Galeev, A.A.; Sudan, R., 1984.

[28] M. Brittnacher, K. B. Quest, and H. Karimabadi. A new approach to the linear theory of singlespecies tearing in two-dimensional quasi-neutral sheets. J. Geophysical Research, 100:3551-3562, March 1995. 